\title{
The C-terminal domain of RNA Pol II helps ensure that editing precedes splicing of the GluR-B transcript
}

\author{
KICKI RYMAN, ${ }^{1}$ NOVA FONG, ${ }^{2}$ EVA BRATT, ${ }^{1}$ DAVID L. BENTLEY, ${ }^{2}$ and MARIE ÖHMAN ${ }^{1}$ \\ ${ }^{1}$ Department of Molecular Biology and Functional Genomics, Stockholm University, S-106 91 Stockholm, Sweden \\ ${ }^{2}$ Department of Biochemistry and Molecular Genetics, University of Colorado Health Sciences Center, Aurora, Colorado 80045, USA
}

\begin{abstract}
The C-terminal domain (CTD) of the large subunit of RNA polymerase II (Pol II) influences many steps in the synthesis of an mRNA and helps control the final destiny of the mature transcript. ADAR2 edits RNA by converting adenosine to inosine within double-stranded or structured RNA. Site-selective A-to-I editing often occurs at sites near exon/intron borders, where it depends on intronic sequences for substrate recognition. It is therefore essential that editing precedes splicing. We have investigated whether there is coordination between ADAR2 editing and splicing of the GluR-B pre-mRNA. We show that the CTD is required for efficient editing at the $R / G$ site one base upstream of a $5^{\prime}$-splice site. The results suggest that the CTD enhances editing at the $\mathrm{R} / \mathrm{G}$ site by preventing premature splicing that would remove the intronic recognition sites for ADAR2. Editing at the GluR-B Q/R site, 24 bases upstream of the intron $115^{\prime}$-splice site, stimulates splicing at this intron. Furthermore, unlike previously studied introns, the CTD actually inhibits excision of intron 11, which includes the complementary recognition sequences for the $Q / R$ editing site. In summary, these results show that the CTD and ADAR2 function together to enforce the order of events that allows editing to precede splicing, and they furthermore suggest a new role for the CTD as a coordinator of two interdependent pre-mRNA processing events.
\end{abstract}

Keywords: RNA editing; ADAR; RNA polymerase II; CTD; RNA processing

\section{INTRODUCTION}

RNA editing by adenosine deamination is the most common type of editing in metazoans. Enzymes that catalyze the reaction of adenosine (A) to inosine (I) base modification are called ADARs, adenosine deaminases that act on RNA (Bass et al. 1997). In mammals, two active ADAR editing enzymes have been characterized, ADAR1 and ADAR2, with somewhat different substrate specificity (Higuchi et al. 2000; Hartner et al. 2004; Wang et al. 2004). ADAR editing has been shown to occur with low selectivity on completely double-stranded RNA (dsRNA) or selectively on structured RNA, interrupted by bulges or loops (for review, see Bass 2002). Most sites for selective editing have been found in mRNAs expressed in the central nervous system in mammals, Drosophila, and squid. Adenosines are edited to inosines that are read as guanosines by the splicing and translation machineries. ADAR

Reprint requests to: Marie Öhman, Department of Molecular Biology and Functional Genomics, Stockholm University, S-106 91 Stockholm, Sweden; e-mail: marie.ohman@molbio.su.se; fax: +46 (8) 166488.

Article published online ahead of print. Article and publication date are at http://www.rnajournal.org/cgi/doi/10.1261/rna.404407. editing can therefore result in codon changes (for review, see Keegan et al. 2001; Bass 2002; Maas et al. 2003) or formation of novel splice acceptor/donor sites with a potential to increase the repertoire of the proteome (Rueter et al. 1999; Beghini et al. 2000; Athanasiadis et al. 2004). The most prominent substrates subjected to A-to-I editing in mammals are pre-mRNAs coding for glutamate and serotonin receptors (Sommer et al. 1991; Burns et al. 1997). ADAR2 can also edit its own pre-mRNA, creating an alternative $3^{\prime}$-splice site (Rueter et al. 1999). This has been suggested to function as an autoregulatory loop to control ADAR2 editing activity. The glutamate receptor subunit B (GluR-B) of the AMPA ( $\alpha$-amino-3-hydroxy-5-methyl-4isoxazole propionate) receptor is edited at two sites within the coding sequence (Fig. 1A). Editing of GluR-B results in altered receptor properties, changing ion channel permeability or recovery after desensitization (Hume et al. 1991; Burnashev et al. 1992; Lomeli et al. 1994). The Q/R site is edited to nearly $100 \%$, giving rise to a codon change from glutamine $(\mathrm{Q})$ to arginine $(\mathrm{R})$ in exon 11 . In exon 13, the edited $R / G$ site causes an arginine to glycine $(G)$ codon change that is developmentally regulated. The dsRNA structure required for ADAR editing at these sites is formed by 
A

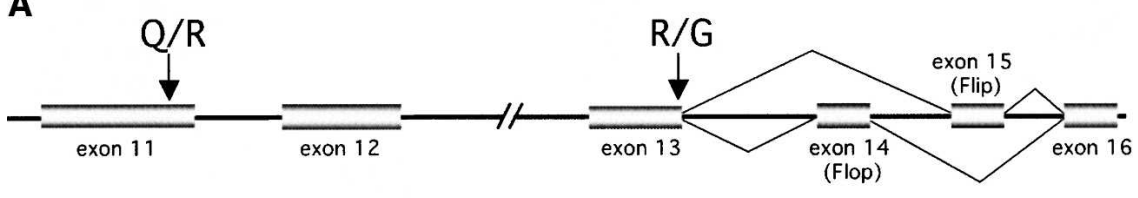

B

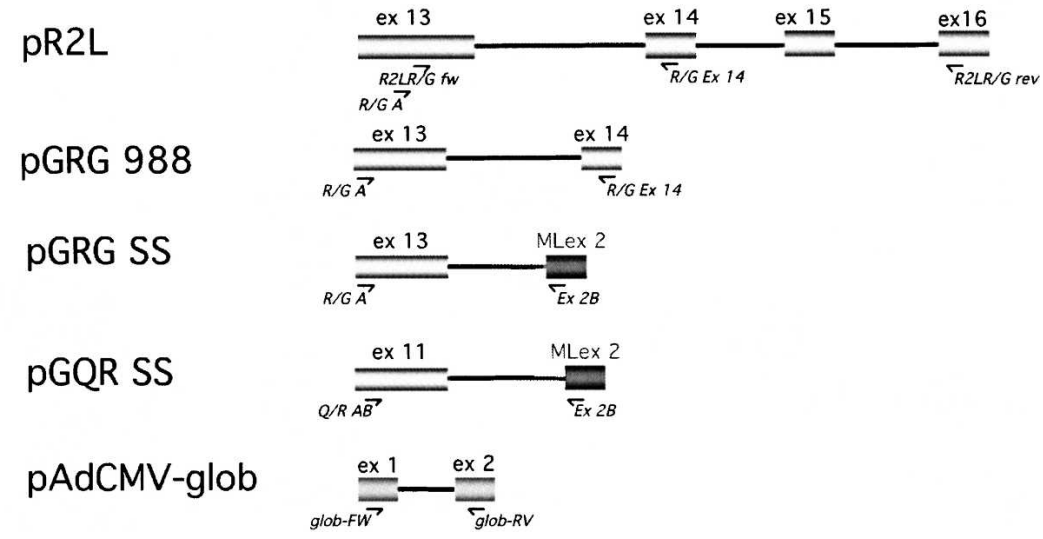

FIGURE 1. (A) Editing and splicing of the GluR-B pre-mRNA from exon 11 through exon 16. (Gray bars) Exons, (lines) introns; not drawn to scale. (Arrows) The $\mathrm{Q} / \mathrm{R}$ and $\mathrm{R} / \mathrm{G}$ editing sites. Alternative splicing of exons 14 and 15 is indicated. (B) Editing/splicing reporter constructs used in the study. The pR2L minigene construct contains the natural GluR-B gene from exon 13 through exon 16. The GRG 988 construct contains a full-length intron 13, flanked by exons 13 and 14. In the GRG SS construct, exon 13 and the part of intron 13 required for R/G editing of GluR-B is fused to part of intron 1, the $3^{\prime}$-splice site, and exon 2 (MLex 2) from a modified adenovirus major late transcript. The GQR SS construct contains the R/G editing sequence fused to the adenovirus MLex2 $3^{\prime}$-splice site. The $\beta$-globin reporter pAdCMV-glob is represented by exon 1 through exon 2 of the rabbit $\beta$-globin pre-mRNA. (Arrows) Positions of primers used for RT-PCR.

the presence of an inverted repeat, located in the downstream introns. It is common that sites of selective editing are in the proximity of splice sites and that the editing event is dependent on intron sequence. In these cases editing must precede splicing, and it is possible that this sequence of events is enforced by a specific regulatory mechanism.

In recent years, considerable evidence has accumulated showing that RNA processing by capping, splicing, and cleavage/polyadenylation is cotranscriptional. The C-terminal domain (CTD) of the largest subunit of RNA polymerase II (RNA Pol II) has been proposed to be the main coordinator of the RNA processing events (for review, see Maniatis and Reed 2002; Kornblihtt et al. 2004; Zorio and Bentley 2004). A reversible phosphorylation of Ser 2 and Ser5 in the CTD repeats occurs during the transcriptional cycle (Dahmus 1996; Palancade and Bensaude 2003). The sequence of the CTD with the consensus heptad repeat element, YSPTSPS, is highly conserved among eukaryotes. The number of repeats differs between species, with 26 heptads in yeast and 52 repeats in humans. Truncation of the CTD has been shown to reduce the efficiency of capping, splicing, and 3'end formation (Cho et al. 1997; McCracken et al. 1997a,b). However, 22-27 tandem heptads of either conserved or divergent repeats are sufficient for efficient splicing and 3'- end formation (Ryan et al. 2002; Fong et al. 2003; Rosonina and Blencowe 2004). A unique 10-residue C-terminal motif, ISPDDSDEEN, is essential for the stability of the large subunit of RNAPII (Rpb1) (Chapman et al. 2004). Little is known about the mechanism for how splicing is dependent on the CTD during transcription in vivo. Nevertheless, since A-to-I editing often occurs prior to splicing, it follows that editing must also be cotranscriptional. Moreover, in a recent report we showed that the Adar2 pre-mRNA is dependent on the CTD for efficient editing and subsequent alternative splicing (Laurencikiene et al. 2006).

In this study we present evidence for a role of the CTD in determining the temporal sequence of splicing and editing by ADAR2. We demonstrate that the CTD is required for efficient editing in a situation where sites of editing and splicing are in close proximity to each other, at the $\mathrm{R} / \mathrm{G}$ editing site in the GluR-B gene. Further, at the Q/R site, where the position of editing is more distant from the splice donor site, the CTD has little effect on editing but regulates the splicing. Our results suggest that the CTD serves to coordinate cotranscriptional splicing and editing by preventing premature splicing at sites that are substrates for the editing enzyme ADAR2.

\section{RESULTS}

\section{Deletion of the CTD inhibits editing at the R/G site}

The R/G editing site is situated only 1 nucleotide (nt) upstream of the exon 13/intron 13 border of Glutamate receptor transcript $\mathrm{B}$. We initially examined the effect of the CTD on editing at this site, independent of splicing, using the reporter construct GRG988 in which splicing of intron 13 is very inefficient as determined by RT-PCR (Fig. 2A). GRG 988 contains exon 13, including the R/G site, full-length intron 13, and exon 14 (flop) driven by the CMV promoter (Fig. 1B). This reporter construct was transiently transfected into HEK 293 cells together with expression vectors for Flag-tagged ADAR2 and wild-type (WT) full-length $\alpha$-amanitin-resistant RNA Pol II large subunit, Rpb1WT, or Rpb1 1 CTD lacking the entire Cterminal domain. By inhibiting the endogenously expressed Pol II with $\alpha$-amanitin, the majority of the steady-state pool of RNAs was synthesized by RNA Pol II with the $\alpha$-amanitin-resistant subunit (Gerber et al. 1995). To 
A

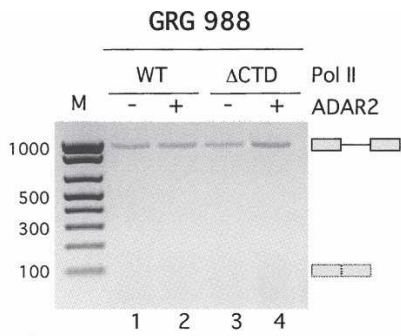

B

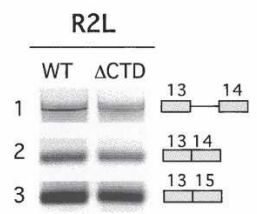

C
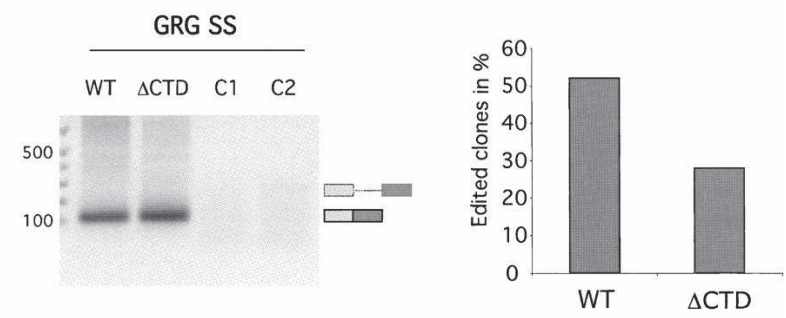

D

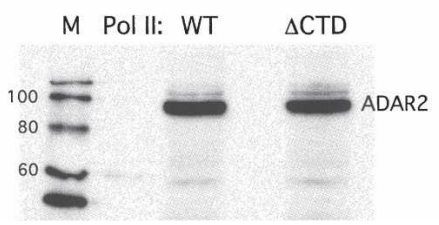

FIGURE 2. Editing and splicing of $\mathrm{R} / \mathrm{G}$ editing reporter constructs. (A) Splicing of GRG 988 analyzed by RT-PCR. The size of the product from the GRG 988 pre-mRNA is $962 \mathrm{nt}$, and the size predicted for the mRNA is $106 \mathrm{nt}$ (indicated to the right of the gel). Transcripts derived from WT or $\triangle$ CTD Pol II transcription are as indicated. Cotransfection of the ADAR2 expression vector is indicated with $(+)$ or $(-)$, and the size in base pairs of bands in the size marker $(\mathrm{M})$ is indicated on the left. (Right) Editing of the GRG 988 pre-mRNA was analyzed by sequence determination of the RT-PCR products. (Arrows) The R/G site sequence. $(B)$ Splicing of the R2L transcript in the presence of ADAR2. Bands 1 and 2 represent products from exon 13- and exon 14-specific primers, and band 3 represents the mRNA product from a PCR using exon 13- and exon 16-specific primers. (Right) Editing of the R2L transcript (band 3) from WT and $\triangle$ CTD Pol II transcription analyzed by sequence determination. (Arrows) The R/G site sequence. (C) Splicing of GRG SS analyzed by RT-PCR. Expected size of the products from pre-mRNA $(260 \mathrm{nt})$ and mRNA $(116 \mathrm{nt})$ are indicated on the right. Controls where either reverse transcriptase was omitted (C1) or no template was added to the PCR reaction (C2) are shown. (Right) Sequence analysis to detect editing at the R/G site of individual clones with RT-PCR products from GRG SS transcripts. Twentyeight percent were edited when transcribed by Pol II $\Delta$ CTD ( $\mathrm{n}=$ 18), whereas $52 \%$ were edited when transcribed by wild-type pol II $(\mathrm{n}=27) .(D)$ Western blot of transient expression of rADAR2 in $\alpha$-amanitin-treated 293 cells transfected with $\alpha$-amanitin-resistant WT or $\triangle$ CTD RNA Pol II. Sizes of bands in lane 1 (M) are in kilodaltons. ensure that ADAR2 expression was not affected by coexpression of Pol II $\triangle \mathrm{CTD}$, a Western blot analysis using a Flag-tag antibody was made to determine the amount of transiently expressed ADAR2. As shown in Figure 2D, the amount of expressed ADAR2 was identical in cells transfected with a WT Pol II and a Pol II $\triangle$ CTD.

In accordance with our previous results (Bratt and Öhman 2003), intron 13 in the GRG988 reporter was not efficiently spliced regardless of whether the CTD was present (Fig. 2A, left). On the other hand, the R/G site was efficiently edited independent of splicing when the gene was transcribed by either WT Pol II or Pol II $\triangle$ CTD (Fig. 2A, right). Editing is seen as a $G$ peak in the chromatogram. This result therefore indicates that the efficiency of the editing event per se is not influenced by the CTD under conditions where splicing does not occur and therefore cannot interfere with editing.

A different minigene, R2L, was used to study editing at the GluR-B R/G site under conditions where intron 13 is efficiently spliced. The R2L construct containing exons 1316 (Fig. 1B) with intervening introns was cotransfected with a WT or $\triangle$ CTD expression vector for $\alpha$-amanitinresistant Pol II and the expression vector for ADAR2. The GluR-B exon 13 is alternatively spliced to exons 14 and 15 (Flop and Flip). Primers in exons 13 and 14 were used to amplify pre-mRNA and the mRNA spliced to exon 14. The efficiency of R2L splicing appeared to be similar for wildtype and $\triangle \mathrm{CTD}$ Pol II (Fig. 2B, bands 1,2). Primers in exons 13 and 16 were used for RT-PCR so that all alternative splice products were amplified, and editing at the $R / G$ site in exon 13 was assayed by sequencing. A prominent negative effect on $\mathrm{R} / \mathrm{G}$ editing was detected when the RNA was transcribed by the CTD deletion mutant $(\triangle C T D)$. The major peak corresponded to an adenosine at the $\mathrm{R} / \mathrm{G}$ site in the chromatogram, indicating that most of the mRNA transcripts were not edited (Fig. 2B). In contrast, processed mRNA transcribed by the RNA Pol II with an intact CTD (WT) allowed efficient editing, seen as a major $\mathrm{G}$ peak (Fig. 2B). The sequence analysis also revealed that the major mRNA product is alternatively spliced from exon 13 to exon 15 (Fig. 2B, band 3).

To ensure that the CTD effect on editing is not due to an indirect effect on alternative splicing, we replaced the natural $3^{\prime}$-splice site of intron 13 with a strong $3^{\prime}$-splice site from the adenovirus major late transcript (GRG SS, Fig. 1B). The GRG SS construct was cotransfected with a WT or $\Delta$ CTD expression vector for $\alpha$-amanitin-resistant Pol II and the expression vector for ADAR2 as above. Unlike several other introns studied previously but similar to R2L, splicing of the GRG SS hybrid intron was not markedly inhibited by a deletion of the CTD (Fig. 2C, left). To investigate whether ADAR2 editing of the GRG SS construct was dependent on the CTD, the RT-PCR products corresponding to spliced transcripts were cloned and sequenced. Editing at the R/G site decreased from $52 \%$ to $28 \%$ in the absence of the CTD (Fig. $2 \mathrm{C}$, right). 
We conclude that the CTD does not influence editing at the GluR-B R/G site in the absence of splicing, but when splicing does occur and can compete with editing, then the CTD is important to permit efficient editing.

\section{Editing at the $Q / R$ site and subsequent splicing is coordinated by the CTD}

The Q/R editing site in GluR-B is situated 24 nt upstream of the $5^{\prime}$-splice site in intron 11 (Fig. 1A). We investigated whether the CTD is also required to coordinate editing and splicing at this site. For these experiments we used a reporter construct, pGQR SS (Fig. 1B), that comprises the $\mathrm{Q} / \mathrm{R}$ editing site in exon 11 and part of intron 11 that includes the editing complementary sequence fused to the strong adenovirus $3^{\prime}$-splice site. The pGQR SS reporter was cotransfected with Rpb1 WT or Rpb1 $\Delta$ CTD expression vector into HEK 293 cells, and RNA from $\alpha$-amanitintreated cells was analyzed by RT-PCR. These experiments showed a slight negative effect of CTD deletion on $\mathrm{Q} / \mathrm{R}$ editing compared with WT $\alpha$-amanitin-resistant Pol II (Fig. 3A). A dual A and G peak at the Q/R site can be seen in the chromatogram upon transcription from the $\Delta \mathrm{CTD}$ Pol II, while a single $G$ peak is seen during transcription by the WT RNA polymerase.

The efficiency of splicing of the chimeric GluR-B intron 11 in the GQR SS reporter gene was determined by RTPCR on total RNA using exon-specific primers. Surprisingly, when the gene was transcribed by the $\Delta$ CTD Pol II, splicing was significantly more efficient than when it was transcribed by the WT Pol II (Fig. 3B, lanes 3-6 and graph). As a control, WT or $\Delta$ CTD Pol II was cotransfected with an exon 1 -intron 1 -exon 2 reporter of the rabbit $\beta$-globin gene. Consistent with previous results (McCracken et al. 1997b), no splicing of the $\beta$-globin reporter was observed when it was transcribed by Pol II lacking the CTD (Fig. 3B, lanes 1,2). Splicing of the GQR SS reporter transcripts was enhanced by transient expression of ADAR2 in the presence of WT Pol II but not with Pol II $\Delta$ CTD (Fig. 3B, cf. lanes 3,4 and 5,6). These results were confirmed using an RNase protection assay on the same reporter construct (data not shown).

We conclude from these experiments that the CTD positively affects editing and negatively affects splicing of the GQR SS transcript. Furthermore, ADAR2 facilitates excision of the GQR SS chimeric intron, but only in presence of the CTD.

\section{Editing at the $Q / R$ site upstream of the 5 '-splice site enhances splicing}

To investigate whether the enhancement of GQR SS splicing by ADAR2 requires enzyme activity, a mutation, E396A, was introduced in the active site (Macbeth et al. 2005). The E396A mutant protein was expressed at similar
A

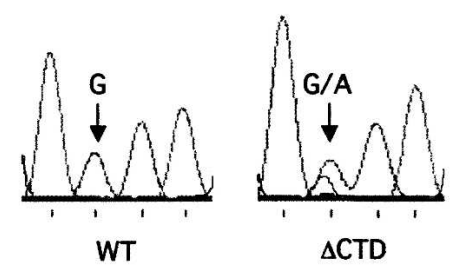

B
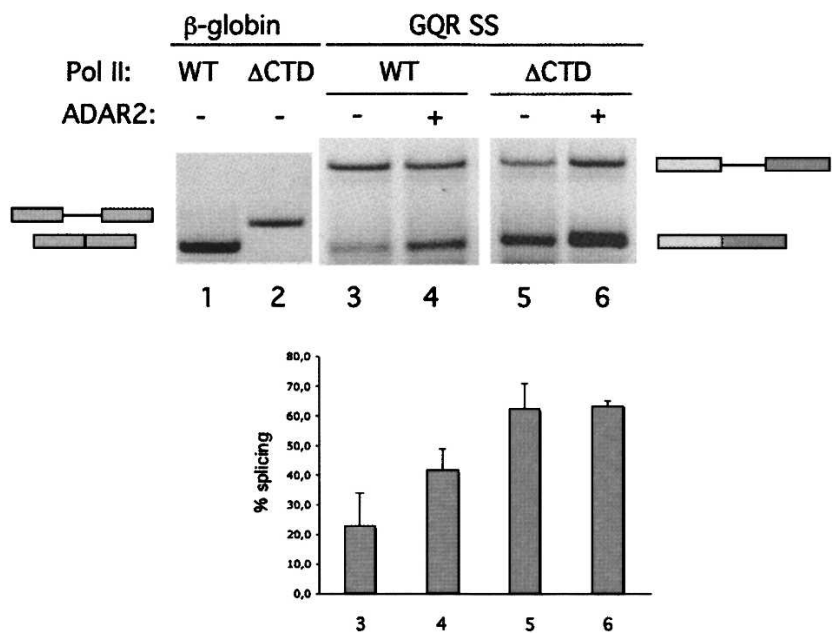

FIGURE 3. Editing and splicing of the GQR SS reporter construct transcribed by $\alpha$-amanitin-resistant RNA polymerases. (A) Sequence analysis of RT-PCR amplification products from GQR SS transcripts. (Arrow) The $\mathrm{Q} / \mathrm{R}$ editing site. The reporter was transcribed by the WT RNA Pol II (WT) or $\Delta$ CTD as indicated. (B) Splicing of the $\beta$-globin and GQR SS reporters transcribed by WT or $\triangle$ CTD RNA Pol II as indicated. Cotransfection of the ADAR2 expression vector is indicated by $(+)$ or $(-)$. (Below) The average percentage of splicing based on three independent experiments. According to Student's t-test statistical analysis the difference in splicing efficiency \pm ADAR2 is significant $(P=0.02)$ as well as the difference \pm CTD $(P=0.03)$. Numbers below the graph correspond to the lanes above.

levels to WT rADAR2 as determined by Western blot (Fig. 4B). No editing at the Q/R site of GluR-B was observed after transient transfection of the E396A mutant construct (data not shown). Further, the E396A mutant protein was not able to induce splicing of the GQR SS transcript in HEK 293 cells (Fig. 4A, lane 4). In fact, we observed an apparent dominant negative effect of the E396A mutant on intron 11 splicing (Fig. 4A, lanes 3,4). This result indicates that ADAR catalysis is required for enhanced splicing of intron 11. To determine if it is the presence of an active enzyme or the A-to-I editing event itself that enhances splicing, the GQR SS reporter was mutated by introducing an $A$ to $G$ substitution at the $Q / R$ site to mimic editing (GQR SS-G). This point mutation enhanced splicing relative to the WT construct similar to the effect seen after ADAR2 expression. This result suggests that it is the change in sequence from A-to-I at position -25, upstream of the 5 '-splice site that stimulates splicing (Fig. 5A). Expression of ADAR2 did not enhance splicing of this substrate 


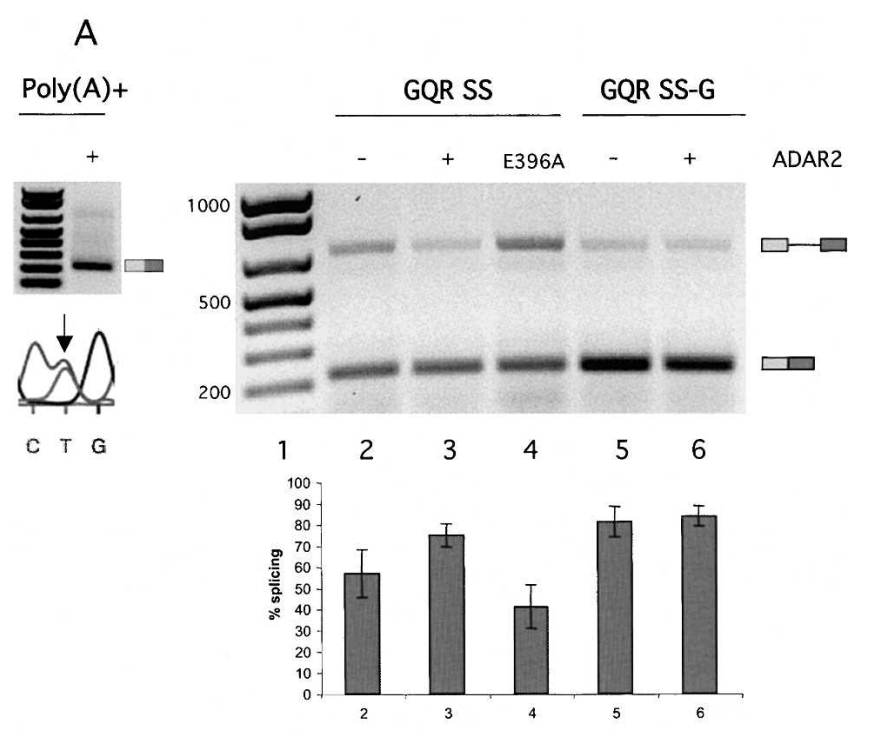

B

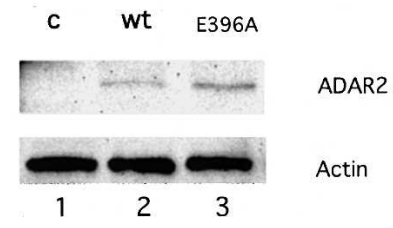

FIGURE 4. Editing and splicing of the pre-edited GQR SS-G premRNA. (A) (Right) Splicing of the GQR SS and GQR SS-G reporter transcripts analyzed by RT-PCR. The ADAR2 expression vector or the mutant E396A was cotransfected as indicated. (Below) The average percentage of splicing based on three independent experiments. Numbers below the graph corresponds to the lanes above. (Left, top) Cytoplasmic poly(A)+ GQR SS RNA analyzed by RT-PCR; (left, bottom) sequence analysis of the RT-PCR product from the GQR SS transcript above using the reverse primer TA10 exon2B. (Arrow) The $\mathrm{Q} / \mathrm{R}$ editing site. Editing is seen as a dual C/T peak in the chromatogram, with the $C$ peak representing inosine. $(B)$ Western blot showing the expression level of rADAR2 protein (lane 2) relative to the E396A mutant (lane 3). (Lane 1) Cells were transfected with an empty vector as a control. The level of ADAR2 was normalized to the expression of actin as indicated.

further; indicating that editing at the $\mathrm{Q} / \mathrm{R}$ site is the main cause of increased splicing efficiency at this intron. To ensure that the transcripts made during transient transfections are stable, properly processed, and transported to the cytoplasm, cytoplasmic RNA was extracted and purified on an oligo dT column. As seen on the left in Figure 4A, the cytoplasmic GRG SS transcript is spliced, polyadenylated, and edited (seen as a $\mathrm{C} / \mathrm{T}$ peak in the chromatogram).

\section{DISCUSSION}

Selectively edited A-to-I sites are often located near exon/ intron borders, and editing often depends upon intronic sequences for substrate recognition. Editing must therefore happen prior to splicing. This can be achieved if ADAR binding has priority over and/or stalls the splicing machin- ery. Editing and splicing are coordinated events in the R/G editing/splicing reporters (R2L and GRG SS) when transcribed by RNA Pol II, while they are competing events in vitro on a substrate transcribed by T7 RNA polymerase (Bratt and Öhman 2003). Further, in a recent report we showed that editing and subsequent alternative splicing of the ADAR2 pre-mRNA requires coordination by the CTD of Pol II to be efficient (Laurencikiene et al. 2006). However, the exact mechanism for how the two RNA processing events are coordinated is not fully elucidated.

In this study, we investigated the role of the CTD in sequential A-to-I editing and pre-mRNA splicing in two cases when sites of editing are close to $5^{\prime}$-splice sites. As model substrates we used reporter constructs based on the $\mathrm{R} / \mathrm{G}$ and the $\mathrm{Q} / \mathrm{R}$ editing sites in the GluR-B pre-mRNA. CTD deletion did not affect editing of the GRG 988 reporter, which is poorly spliced regardless of the CTD. We conclude that editing per se is not dependent on the CTD (Fig. 2A). On the other hand, in a transcript with a more efficient splicing event, as in the R2L or the GRG SS construct (Fig. 2B,C), then the CTD has an important positive effect on the extent of editing. The GluR-B R/G editing site is located only $1 \mathrm{nt}$ upstream of the $5^{\prime}$-splice site, and therefore U1 snRNP or other spliceosomal components could compete with ADAR2 for binding at this position. In vivo, however, a mechanism appears to exist to ensure that cotranscriptional editing and splicing

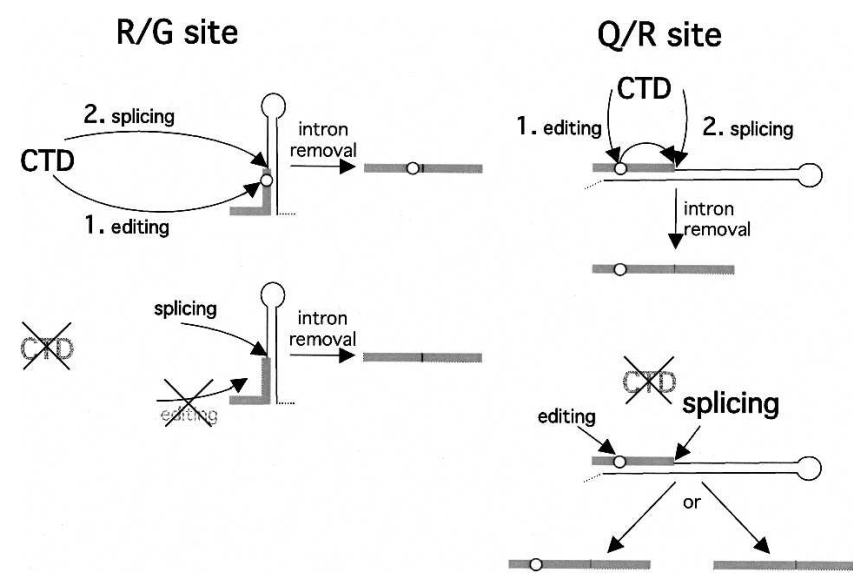

FIGURE 5. Models for the coordination of editing and splicing by the transcription machinery. ( $R / G$ site) Editing and splicing of the GluR-B R/G substrate. During transcription in the presence of the $\mathrm{CTD}$, splicing is delayed until after R/G editing. After intron removal, the majority of the mRNAs are therefore edited. In the absence of the CTD, there is competition between splicing and editing that prevents editing. After intron removal, the majority of the mRNAs have escaped editing. (Q/R site) Editing and splicing of the GluR-B Q/R substrate. During transcription in the presence of the CTD, splicing is delayed until after $\mathrm{Q} / \mathrm{R}$ editing. The A-to-I change at the $\mathrm{Q} / \mathrm{R}$ site has a positive effect on splicing. After intron removal, most of the mRNAs are therefore edited. In the absence of the CTD, editing and splicing occur independently. After intron removal, mRNAs will have escaped editing, although a subset of the molecules will be edited. 
occur sequentially. The more efficiently spliced GRG SS construct is edited to a similar extent as the alternatively spliced R2L substrate. Nevertheless, our results suggest the CTD contributes to the coordination of these processing events by delaying splicing until after editing has occurred. In the absence of the CTD, splicing of the GRG SS reporter is still very efficient but editing is inhibited (Fig. 2C), possibly because ADAR2 is out-competed by spliceosome assembly at the $5^{\prime}$-splice site (Fig. 5, left). This is also true for the R2L reporter. A delay in splicing until after editing might not necessarily be observed as less efficient splicing as it is the steady-state pool of transcripts that are analyzed. Nevertheless, in absence of the CTD it is clear that many spliced mRNAs have escaped editing as is shown in Figure 2, B and C.

Editing at the $\mathrm{Q} / \mathrm{R}$ site occurs $24 \mathrm{nt}$ upstream of the intron $115^{\prime}$-splice site. As observed at the R/G site, the CTD is also required to coordinate editing and splicing at the $\mathrm{Q} / \mathrm{R}$ site, probably by delaying splicing until after editing is complete (Fig. 5, right). Transcription by an RNA polymerase lacking the CTD enhances splicing of the GQR SS reporter transcript (Fig. 3), strongly suggesting that the CTD normally acts to restrict premature splicing at this site. This is in line with a recent report showing that the CTD can promote exon skipping by recruiting SRp20 and thereby induce alternative splicing (de la Mata and Kornblihtt 2006). A unique feature of the $Q / R$ substrate is that editing leads to a more efficient splicing, but only in the presence of the CTD (Figs. 3, 4). Even though the Q/R site (in bold) is included in a putative mouse exonic splicing enhancer (ESE) element (AGCAAG) (Fairbrother et al. 2004), we have no explanation for this effect, since editing at the $\mathrm{Q} / \mathrm{R}$ site disrupts the ESE. However, our results are consistent with a previous report showing that splicing of intron 11 of the GluR-B transcripts is reduced in Adar2 null mice that have low levels of editing (Higuchi et al. 2000). Moreover, in Drosophila there is a strong correlation between alternative splice-site selection and editing status in a gene where the editing site is located upstream of alternative splice sites (Agrawal and Stormo 2005). In contrast, however, no correlation between alternative splicing and editing was observed when the editing site was located downstream of alternative splice sites. In summary, our results suggest that splicing and editing can influence each other and that the CTD helps restrain splicing to facilitate editing at the $\mathrm{Q} / \mathrm{R}$ site.

At present there is no compelling evidence for a direct protein-protein interaction between ADAR2 and the Pol II CTD. Some splicing-related factors bind directly to the phosphorylated CTD, including Prp40, CA150, and proteins with SR domains, and it is possible that these factors contribute to the CTD-dependent delay in splicing at GluR-B introns 11 and 13 (Yuryev et al. 1996; Morris and Greenleaf 2000; Goldstrohm et al. 2001; Katsarou et al. 2005). It is also possible that direct or indirect association of the $5^{\prime}$ exon RNA with the CTD could contribute to a delay in assembly of the spliceosome that facilitates editing. Consistent with this idea, it has recently been suggested that exons are tethered by Pol II (Dye et al. 2006). Moreover, it is possible that disruption of the coupling between capping, splicing, and cleavage/polyadelylation when the CTD is deleted could influence editing.

In summary, our results show that editing and splicing of the glutamate receptor $\mathrm{B}$ are cotranscriptional events that require the Pol II CTD in order to occur sequentially (Fig. 5). At both edited sites (R/G and Q/R), splicing is stalled by the CTD to ensure that the order of events is first editing and then splicing. In the absence of a functional CTD, this coordinating function is disrupted, resulting in premature splicing that prevents editing.

\section{MATERIALS AND METHODS}

\section{Plasmids and deoxyoligonucleotides}

All mammalian expression vectors used in this study are based on the pcDNA3 vector (Invitrogen), except the vectors expressing RNA polymerase II (see below). The editing/splicing reporter sequences were cloned into the multiple cloning site of this vector, situated downstream of the CMV promoter and upstream of the $\mathrm{BGH}$ poly(A) site. Constructs containing the R/G editing site from the rat GluR-B gene are: pcDNA3/R2L (pR2L), pcDNA3Flag/GRG 988 (pGRG 988), and pcDNA3Flag/GRG SS (pGRG SS). The pR2L plasmid was kindly provided by the laboratory of Jørgen Kjems, University of Aarhus, Denmark. In the pR2L plasmid, a WT $g l u R-B$ sequence from exon 13 to exon 16 was inserted. The GRG 988 reporter contains exons 13 and 14 (flop) with an intervening intron 13, and in pGRG SS, exon 13 and the $5^{\prime}$-splice site of intron 13 were fused upstream of a modified adenovirus acceptor site, followed by the major late exon 2. Both reporters are described by Bratt and Öhman (2003). In the Q/R editing/splicing reporter (pGQR SS), the rat gluR-B sequence including exon 11 and $395 \mathrm{nt}$ of intron 11 was fused to the adenovirus intron branchpoint and acceptor site from the pTA10 vector as above and described by Bratt and Öhman (2003). The pGQR SS-G plasmid was made by site-directed mutagenesis using the pGQR SS vector and the QuikChange XL Site-Directed Mutagenesis Kit (Stratagene) according to the manufacturer's instructions. The primers used to change the A for a $\mathrm{G}$ at the $\mathrm{Q} / \mathrm{R}$ site were " $\mathrm{QRs} \mathrm{dm}$ forw” (5'-tccttgggtgcctttatgcggcaaggatgcgatatttcg-3') and "QRsdm rev" (5'-cgaaaatatcgcatccttgccgcataaaggcacccaagga- $\left.3^{\prime}\right)$. The cDNA encoding rat ADAR2a used in the transient transfections to express ADAR2 was as previously described (Bratt and Öhman 2003). The E396A rADAR2 mutant was obtained by site-directed mutagenesis using the rat ADAR2 expression vector and the QuikChange XL Site-Directed Mutagenesis Kit (Stratagene). The primers used were "sdmfwAtoC" (5'-ctcaatgactgccacgcagcgataatctcccgaaggtcc- 3 ') and “sdmrvTtoG” (5'-ggaccttcgggagattatcgctgcgtggcagtcattgag- 3 ').

Plasmids expressing the $\alpha$-amanitin-resistant large subunit of RNA polymerase II were WT CTD, pATRpb1 (Nguyen et al. 1996), or a polymerase lacking all the heptad repeats of the CTD $(\Delta \mathrm{CTD})$, pATRpb1 $\Delta 0$, as previously described (Fong and Bentley 2001). 
The primers used for reverse transcription (RT) in this work were TA10 exon2B (5'-gagaccgcgaagagtttgtcctgc-3') or random hexamers (Invitrogen). PCR amplification of the cDNA was done using the following primers: for GRG SS, R/G A (5'-ccttgtgacac catgaaagtggg-3') and TA10 exon2B; GRG 988, R/G A and $\mathrm{R} / \mathrm{G}$ exon14 (5'-tttagtactgcgaggttaaccgc- $\left.3^{\prime}\right)$; R2L, R2LR/G fw (5'-gaggaagccttgtgacaccatg- $3^{\prime}$ ) and R2Lexon16 rev (5'-cgttgttcaga ctgagggcac- $3^{\prime}$ ) or R/G A and R/G exon14; and for GQR SS the $\mathrm{Q} / \mathrm{R} \mathrm{AB}\left(5^{\prime}\right.$-tttgcctacattggggtcagtg- $\left.3^{\prime}\right)$ and the TA10 exon2B primer. The pAdCMV-glob plasmid used as a template for the expression of the rabbit exon 1-intron 1-exon $2 \beta$-globin transcript was kindly provided by Göran Akusjärvi, Uppsala University, Sweden. Primers used for RT-PCR to amplify the $\beta$-globin transcript were glob-fw $\left(5^{\prime}\right.$ - cagaatggtgcatctgtccag- $\left.3^{\prime}\right)$ and glob-rv ( $5^{\prime}$-agaccctcactgaaggcagcc- $\left.3^{\prime}\right)$.

\section{Transient transfections and RNA analysis}

HEK 293 cells were transiently transfected with pcDNA3Flag/ rADAR2 or the empty pcDNA3Flag vector and one of the editing/ splicing reporters containing the $\mathrm{Q} / \mathrm{R}$ or $\mathrm{R} / \mathrm{G}$ site, together with an $\alpha$-amanitin-resistant RNA polymerase II (see above) using Lipofectamine 2000 (Invitrogen). A total of $20 \mu \mathrm{g}$ of DNA and $30 \mu \mathrm{L}$ of Lipofectamine 2000 were used in each culture dish $\left(100 \mathrm{~cm}^{2}\right)$; $2.5 \mu \mathrm{g} / \mathrm{mL} \alpha$-amanitin (Sigma) was added to the cells $16 \mathrm{~h}$ posttransfection. Cells were harvested $\sim 64 \mathrm{~h}$ after transfection, and total RNA was extracted from cells using a GenElute mammalian total RNA kit (Sigma).

Editing and splicing were analyzed using RT-PCR. To remove contaminated DNA, the total RNA was treated with DNase I (Sigma) for $15 \mathrm{~min}$ at room temperature. To inactivate the enzyme and make the RNA more accessible to the reverse transcriptase, the RNA was further incubated for $10 \mathrm{~min}$ at $70^{\circ} \mathrm{C}$. First-strand cDNA was synthesized from 1-3 $\mu \mathrm{g}$ of DNase-treated RNA, using M-MLV reverse transcriptase or Superscript III (Invitrogen) according to the manufacturer's instructions. Amplification of cDNAs was made by two-step PCR using Taq polymerase (Clontech) with an annealing temperature of $68^{\circ} \mathrm{C}$. The PCR reaction was tested to be within the linear range at 25 cycles. PCR products were analyzed on a $2 \%$ agarose gel. Splicing efficiency was defined by measuring the intensity of spliced and nonspliced PCR products using BioRad Geldoc equipment and the ImageGauge software.

Cytoplasmic RNA was isolated from transfected cells (see above). Cells growing in 150-mm culture dishes were rinsed twice with ice-cold $1 \times$ PBS, harvested in $1 \mathrm{~mL}$ ice-cold $1 \times \mathrm{PBS}$, and centrifuged $(100 \mathrm{~g})$ for $10 \mathrm{~min}$ at $+4^{\circ}$. The cell pellet was resuspended in $300 \mu \mathrm{L}$ of buffer A (10 mM Hepes [pH 7.9], 10 $\mathrm{mM} \mathrm{KCl}$, and $1.5 \mathrm{mM} \mathrm{MgCl}_{2}, 1 \mathrm{mM} \mathrm{DTT}$ ), and incubated for 15 min on ice. The cells were then homogenized by rapidly pushing the cells through a 23-g needle three times and centrifuged at $+4^{\circ}$ for $20 \mathrm{sec}$ at 12,000g. The supernatant containing the cytoplasmic RNA was transferred to a fresh Eppendorf tube. One milliliter of Trizol Reagent (Invitrogen) was used for RNA isolation according to the manufacturer's instructions.

\section{Analysis of RNA editing}

Sequencing of the products from the RT-PCR reactions was made by MWG (Germany) after agarose gel purification using QIAquick (Qiagen). Editing was quantified by evaluating the peaks in the sequencing chromatogram or the PCR products were cloned into the pGEM-T vector (Promega), and individual clones were sequenced. The primer $\mathrm{Q} / \mathrm{R} \mathrm{AB}$ was used for sequence determination at the $\mathrm{Q} / \mathrm{R}$ editing site. The GluR/G A and the R2LR/G primers were used to sequence the R/G editing site in GRG SS and R2L, respectively.

\section{Western blot}

HEK 293 cells were transiently transfected with pcDNA3/rADAR2 or E396A. When the $\alpha$-amanitin-resistant RNA Pol II expression vector was cotransfected, $\alpha$-amanitin was added $16 \mathrm{~h}$ post-transfection. The cells were harvested, after washing with phosphate buffer saline (PBS), $64 \mathrm{~h}$ post-transfection. The samples were separated on an $8 \%$ SDS-PAGE and electroblotted onto a PVDF membrane (Hybond P, Amersham Bioscience). The membrane was blocked in $10 \%$ dry milk in TBS-Tween (10 mM Tris $\mathrm{pH} 8$, $150 \mathrm{mM} \mathrm{NaCl}$, and $0.05 \%$ Tween-20) for $1 \mathrm{~h}$ at room temperature. The membrane was probed with Anti-Flag antibody, diluted 1:1000 for $1 \mathrm{~h}$ at room temperature or overnight at $+4^{\circ} \mathrm{C}$. After extensive washing in TBS-T, the membrane was probed with horseradishperoxidase-conjugated goat anti-rabbit antibody (Dako) diluted 1:3000 in TBS-T. The antibody was detected using ECL-PLUS according to the manufacturer's instructions (Amersham Bioscience) and developed in a CCD camera (LAS 1000) for 1-10 min.

\section{ACKNOWLEDGMENTS}

We thank Lars Wieslander for fruitful discussions and for critically reading the manuscript. This work was supported by grants from the Swedish Natural Science Research Council and Carl Tryggers Stiftelse. D.B. and N.F. are supported by NIH grant GM58613.

Received March 11, 2007; accepted April 23, 2007.

\section{REFERENCES}

Agrawal, R. and Stormo, G.D. 2005. Editing efficiency of a Drosophila gene correlates with a distant splice site selection. RNA 11: 563566.

Athanasiadis, A., Rich, A., and Maas, S. 2004. Widespread A-to-I RNA editing of Alu-containing mRNAs in the human transcriptome. PLoS Biol. 2: doi: 10.1371/journal.pbio.0020391.

Bass, B.L. 2002. RNA editing by adenosine deaminases that act on RNA. Annu. Rev. Biochem. 71: 817-846.

Bass, B.L., Nishikura, K., Keller, W., Seeburg, P.H., Emeson, R.B., O'Connell, M.A., Samuel, C.E., and Herbert, A. 1997. A standardized nomenclature for adenosine deaminases that act on RNA. RNA 3: 947-949.

Beghini, A., Ripamonti, C.B., Peterlongo, P., Roversi, G., Cairoli, R., Morra, E., and Larizza, L. 2000. RNA hyperediting and alternative splicing of hematopoietic cell phosphatase (PTPN6) gene in acute myeloid leukemia. Hum. Mol. Genet. 9: 2297-2304.

Bratt, E. and Öhman, M. 2003. Coordination of editing and splicing of glutamate receptor pre-mRNA. RNA 9: 309-318.

Burnashev, N., Monyer, H., Seeburg, P.H., and Sakmann, B. 1992. Divalent ion permeability of AMPA receptor channels is dominated by the edited form of a single subunit. Neuron 8: 189-198.

Burns, C.M., Chu, H., Rueter, S.M., Hutchinson, L.K., Canton, H., Sanders-Bush, E., and Emeson, R.B. 1997. Regulation of serotonin-2C receptor G-protein coupling by RNA editing. Nature 387: 303-308. 
Chapman, R.D., Palancade, B., Lang, A., Bensaude, O., and Eick, D. 2004. The last CTD repeat of the mammalian RNA polymerase II large subunit is important for its stability. Nucleic Acids Res. 32: $35-44$.

Cho, E.J., Takagi, T., Moore, C.R., and Buratowski, S. 1997. mRNA capping enzyme is recruited to the transcription complex by phosphorylation of the RNA polymerase II carboxy-terminal domain. Genes \& Dev. 11: 3319-3326.

Dahmus, M.E. 1996. Reversible phosphorylation of the C-terminal domain of RNA polymerase II. J. Biol. Chem. 271: 19009-19012.

Dye, M.J., Gromak, N., and Proudfoot, N.J. 2006. Exon tethering in transcription by RNA polymerase II. Mol. Cell 21: 849-859.

de la Mata, M. and Kornblihtt, A.R. 2006. RNA polymerase II Cterminal domain mediates regulation of alternative splicing by SRp20. Nat. Struct. Mol. Biol. 13: 973-980.

Fairbrother, W.G., Yeo, G.W., Yeh, R., Goldstein, P., Mawson, M., Sharp, P.A., and Burge, C.B. 2004. RESCUE-ESE identifies candidate exonic splicing enhancers in vertebrate exons. Nucleic Acids Res. 32: W187-W190.

Fong, N. and Bentley, D.L. 2001. Capping, splicing, and 3' processing are independently stimulated by RNA polymerase II: Different functions for different segments of the CTD. Genes \& Dev. 15: 1783-1795.

Fong, N., Bird, G., Vigneron, M., and Bentley, D.L. 2003. A 10 residue motif at the C-terminus of the RNA pol II CTD is required for transcription, splicing and $3^{\prime}$ end processing. EMBO J. 22: 42744282.

Gerber, H.P., Hagmann, M., Seipel, K., Georgiev, O., West, M.A., Litingtung, Y., Schaffner, W., and Corden, J.L. 1995. RNA polymerase II C-terminal domain required for enhancer-driven transcription. Nature 374: 660-662.

Goldstrohm, A.C., Albrecht, T.R., Sune, C., Bedford, M.T., and Garcia-Blanco, M.A. 2001. The transcription elongation factor CA150 interacts with RNA polymerase II and the pre-mRNA splicing factor SF1. Mol. Cell. Biol. 21: 7617-7628.

Hartner, J.C., Schmittwolf, C., Kispert, A., Muller, A.M., Higuchi, M., and Seeburg, P.H. 2004. Liver disintegration in the mouse embryo caused by deficiency in the RNA-editing enzyme ADAR1. J. Biol. Chem. 279: 4894-4902.

Higuchi, M., Maas, S., Single, F.N., Hartner, J., Rozov, A., Burnashev, N., Feldmeyer, D., Sprengel, R., and Seeburg, P.H. 2000. Point mutation in an AMPA receptor gene rescues lethality in mice deficient in the RNA-editing enzyme ADAR2. Nature 406: $78-81$.

Hume, R.I., Dingledine, R., and Heinemann, S.F. 1991. Identification of a site in glutamate receptor subunits that controls calcium permeability. Science 253: 1028-1031.

Katsarou, M.E., Papakyriakou, A., Katsaros, N., and Scorilas, A. 2005. Expression of the C-terminal domain of novel human SR-A1 protein: Interaction with the CTD domain of RNA polymerase II. Biochem. Biophys. Res. Commun. 334: 61-68.

Keegan, L.P., Gallo, A., and O'Connell, M.A. 2001. The many roles of an RNA editor. Nat. Rev. Genet. 2: 869-878.

Kornblihtt, A.R., de la Mata, M., Fededa, J.P., Munoz, M.J., and Nogues, G. 2004. Multiple links between transcription and splicing. RNA 10: 1489-1498.
Laurencikiene, J., Kallman, A.M., Fong, N., Bentley, D.L., and Ohman, M. 2006. RNA editing and alternative splicing: The importance of co-transcriptional coordination. EMBO Rep. 7: 303-307.

Lomeli, H., Mosbacher, J., Melcher, T., Hoger, T., Geiger, J.R., Kuner, T., Monyer, H., Higuchi, M., Bach, A., and Seeburg, P.H. 1994. Control of kinetic properties of AMPA receptor channels by nuclear RNA editing. Science 266: 1709-1713.

Maas, S., Rich, A., and Nishikura, K. 2003. A-to-I RNA editing: Recent news and residual mysteries. J. Biol. Chem. 278: 1391-1394.

Macbeth, M.R., Schubert, H.L., Vandemark, A.P., Lingam, A.T., Hill, C.P., and Bass, B.L. 2005. Inositol hexakisphosphate is bound in the ADAR2 core and required for RNA editing. Science 309: 1534-1539.

Maniatis, T. and Reed, R. 2002. An extensive network of coupling among gene expression machines. Nature 416: 499-506.

McCracken, S., Fong, N., Rosonina, E., Yankulov, K., Brothers, G., Siderovski, D., Hessel, A., Foster, S., Shuman, S., and Bentley, D.L. 1997a. 5'-Capping enzymes are targeted to pre-mRNA by binding to the phosphorylated carboxy-terminal domain of RNA polymerase II. Genes \& Dev. 11: 3306-3318.

McCracken, S., Fong, N., Yankulov, K., Ballantyne, S., Pan, G., Greenblatt, J., Patterson, S.D., Wickens, M., and Bentley, D.L. 1997b. The C-terminal domain of RNA polymerase II couples mRNA processing to transcription. Nature 385: 357-361.

Morris, D.P. and Greenleaf, A.L. 2000. The splicing factor, Prp40, binds the phosphorylated carboxyl-terminal domain of RNA polymerase II. J. Biol. Chem. 275: 39935-39943.

Nguyen, V.T., Giannoni, F., Dubois, M.-F., Seo, S.-J., Vigneron, M., Kedinger, C., and Bensaude, O. 1996. In vivo degradation of RNA polymerase II largest subunit triggered by $\alpha$-amanitin. Nucleic Acids Res. 15: 2924-2929.

Palancade, B. and Bensaude, O. 2003. Investigating RNA polymerase II carboxyl-terminal domain (CTD) phosphorylation. Eur. J. Biochem. 270: 3859-3870.

Rosonina, E. and Blencowe, B.J. 2004. Analysis of the requirement for RNA polymerase II CTD heptapeptide repeats in pre-mRNA splicing and 3'-end cleavage. RNA 10: 581-589.

Rueter, S.M., Dawson, T.R., and Emeson, R.B. 1999. Regulation of alternative splicing by RNA editing. Nature 399: 75-80.

Ryan, K., Murthy, K.G., Kaneko, S., and Manley, J.L. 2002. Requirements of the RNA polymerase II C-terminal domain for reconstituting pre-mRNA 3' cleavage. Mol. Cell. Biol. 22: 1684-1692.

Sommer, B., Kohler, M., Sprengel, R., and Seeburg, P.H. 1991. RNA editing in brain controls a determinant of ion flow in glutamategated channels. Cell 67: 11-19.

Wang, Q., Miyakoda, M., Yang, W., Khillan, J., Stachura, D.L., Weiss, M.J., and Nishikura, K. 2004. Stress-induced apoptosis associated with null mutation of ADAR1 RNA editing deaminase gene. J. Biol. Chem. 279: 4952-4961.

Yuryev, A., Patturajan, M., Litingtung, Y., Joshi, R.V., Gentile, C., Gebara, M., and Corden, J.L. 1996. The C-terminal domain of the largest subunit of RNA polymerase II interacts with a novel set of serine/arginine-rich proteins. Proc. Natl. Acad. Sci. 93: 6975-6980.

Zorio, D.A. and Bentley, D.L. 2004. The link between mRNA processing and transcription: Communication works both ways. Exp. Cell Res. 296: 91-97. 

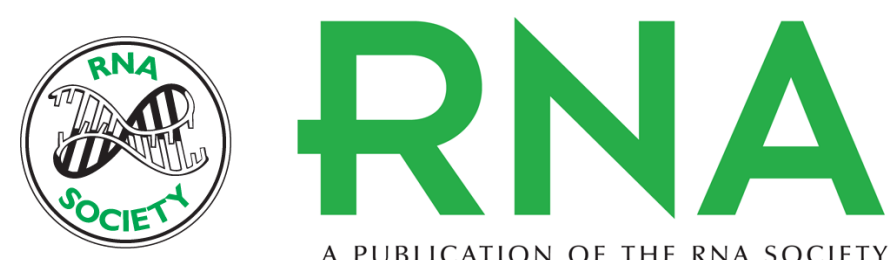

A PUBLICATION OF THE RNA SOCIETY

\section{The C-terminal domain of RNA Pol II helps ensure that editing precedes splicing of the GluR-B transcript}

Kicki Ryman, Nova Fong, Eva Bratt, et al.

RNA 2007 13: 1071-1078 originally published online May 24, 2007

Access the most recent version at doi:10.1261/rna.404407

\section{References This article cites 41 articles, 19 of which can be accessed free at:} http://rnajournal.cshlp.org/content/13/7/1071.full.html\#ref-list-1

\section{License}
Email Alerting Receive free email alerts when new articles cite this article - sign up in the box at the Service top right corner of the article or click here.

To subscribe to $R N A$ go to:

http://rnajournal.cshlp.org/subscriptions 